\title{
Optimal execution strategies in limit order books with general shape functions
}

\author{
Aurélien Alfonsi* \\ CERMICS, projet MATHFI \\ Ecole Nationale des Ponts et Chaussées \\ 6-8 avenue Blaise Pascal \\ Cité Descartes, Champs sur Marne \\ 77455 Marne-la-vallée, France
}

\author{
Antje Fruth \\ Quantitative Products Laboratory \\ Alexanderstr. 5 \\ 10178 Berlin, Germany \\ fruth@math.tu-berlin.de
}

\author{
Alexander Schied* \\ Department of Mathematics, MA 7-4 \\ TU Berlin \\ Strasse des 17. Juni 136 \\ 10623 Berlin, Germany \\ schied@math.tu-berlin.de \\ To appear in Quantitative Finance \\ Submitted September 3, 2007, accepted July 24, 2008 \\ This version: November 20, 2009
}

\begin{abstract}
We consider optimal execution strategies for block market orders placed in a limit order book (LOB). We build on the resilience model proposed by Obizhaeva and Wang (2005) but allow for a general shape of the LOB defined via a given density function. Thus, we can allow for empirically observed LOB shapes and obtain a nonlinear price impact of market orders. We distinguish two possibilities for modeling the resilience of the LOB after a large market order: the exponential recovery of the number of limit orders, i.e., of the volume of the LOB, or the exponential recovery of the bid-ask spread. We consider both of these resilience modes and, in each case, derive explicit optimal execution strategies in discrete time. Applying our results to a block-shaped LOB, we obtain a new closed-form representation for the optimal strategy of a risk-neutral investor, which explicitly solves the recursive scheme given in Obizhaeva and Wang (2005). We also provide some evidence for the robustness of optimal strategies with respect to the choice of the shape function and the resilience-type.
\end{abstract}

${ }^{*}$ Supported by Deutsche Forschungsgemeinschaft through the Research Center MATHEON "Mathematics for key technologies" (FZT 86). 


\section{Introduction.}

A common problem for stock traders consists in unwinding large block orders of shares, which can comprise up to twenty percent of the daily traded volume of shares. Orders of this size create significant impact on the asset price and, to reduce the overall market impact, it is necessary to split them into smaller orders that are subsequently placed throughout a certain time interval. The question at hand is thus to allocate an optimal proportion of the entire order to each individual placement such that the overall price impact is minimized.

Problems of this type were investigated by Bertsimas and Lo [8], Almgren and Chriss [3, 4], Almgren and Lorenz [5], Obizhaeva and Wang [16], and Schied and Schöneborn [18, [19] to mention only a few. For extensions to situations with several competing traders, see [11, [12], [20], and the references therein.

The mathematical formulation of the corresponding optimization problem relies first of all on specifying a stock price model that takes into account the often nonlinear feedback effects resulting from the placement of large orders by a 'large trader'. In the majority of models in the literature, such orders affect the stock price in the following two ways. A first part of the price impact is permanent and forever pushes the price in a certain direction (upward for buy orders, downward for sell orders). The second part, which is usually called the temporary impact, has no duration and only instantaneously affects the trade that has triggered it. It is therefore equivalent to a (possibly nonlinear) penalization by transaction costs. Models of this type underlie the above-mentioned papers [8], [3], 4], [5], [11, [12, and [20]. Also the market impact models described in Bank and Baum [7], Cetin et al. [13], Frey [14], and Frey and Patie [15] fall into that category. While most of these models start with the dynamics of the asset price process as a given fundamental, Obizhaeva and Wang [16] recently proposed a market impact model that derives its dynamics from an underlying model of a limit order book (LOB). In this model, the ask part of the LOB consists of a uniform distribution of shares offered at prices higher than the current best ask price. When the large trader is not active, the mid price of the LOB fluctuates according to the actions of noise traders, and the bid-ask spread remains constant. A buy market order of the large trader, however, consumes a block of shares located immediately to the right of the best ask and thus increase the ask price by a linear proportion of the size of the order. In addition, the LOB will recover from the impact of the buy order, i.e., it will show a certain resilience. The resulting price impact will neither be instantaneous nor entirely permanent but will decay on an exponential scale.

The model from [16] is quite close to descriptions of price impact on LOBs found in empirical studies such as Biais et al. [9], Potters and Bouchaud [17], Bouchaud et al. [10], and Weber and Rosenow [21]. In particular, the existence of a strong resilience effect, which stems from the placement of new limit orders close to the bid-ask spread, seems to be a well established fact, although its quantitative features seem to be the subject of an ongoing discussion.

In this paper, we will pick up the LOB-based market impact model from [16] and generalize it by allowing for a nonuniform price distribution of shares within the LOB. The resulting LOB shape which is nonconstant in the price conforms to empirical observations made in [9, 17, 10, 21]. It also leads completely naturally to a nonlinear price impact of market orders as found in an empirical study by Almgren et al. [6]; see also Almgren [2] and the references therein. In this generalized model, we will also consider the following two distinct possibilities for modeling the resilience of the LOB after a large market order: the exponential recovery 
of the number of limit orders, i.e., of the volume of the LOB (Model 1), or the exponential recovery of the bid-ask spread (Model 2). While one can imagine also other possibilities, we will focus on these two obvious resilience modes. Note that we assume the LOB shape to be constant in time. Having a time-varying LOB shape will be an area of ongoing research.

We do not have a classical permanent price impact in our model for the following reasons: Adding classical permanent impact, which is proportional to the volume traded, would be somewhat artificial in our model. In addition, this would not change optimal strategies as the optimization problem will be exactly the same as without permanent impact. What one would want to have instead is a permanent impact with a sensible meaning in the LOB context. But this would bring substantial difficulties in our derivation of optimal strategies.

After introducing the generalized LOB with its two resilience modes, we consider the problem of optimally executing a buy order for $X_{0}$ shares within a certain time frame $[0, T]$. The focus on buy orders is for the simplicity of the presentation only, completely analogous results hold for sell orders as well. While most other papers, including [16], focus on optimization within the class of deterministic strategies, we will here allow for dynamic updating of trading strategies, that is, we optimize over the larger class of adapted strategies. We will also allow for intermediate sell orders in our strategies. Our main results, Theorem 4.1 and Theorem 5.1, will provide explicit solutions of this problem in Model 1 and Model 2, respectively. Applying our results to a block-shaped LOB, we obtain a new closed-form representation for the corresponding optimal strategy, which explicitly solves the recursive scheme given in [16]. Looking at several examples, we will also find some evidence for the robustness of the optimal strategy. That is the optimal strategies are qualitatively and quantitatively rather insensitive with respect to the choice of the LOB shape. In practice, this means that we can use them even though the LOB is not perfectly calibrated and has a small evolution during the execution strategy.

The model we are using here is time homogeneous: the resilience rate is constant and trading times are equally spaced. By using the techniques introduced in our subsequent paper [1], it is possible to relax these assumptions and to allow for time inhomogeneities and also for linear constraints, at least in block-shaped models.

The method we use in our proofs is different from the approach used in [16]. Instead of using dynamic programming techniques, we will first reduce the model of a full LOB with nontrivial bid-ask spreads to a simplified model, for which the bid-ask spreads have collapsed but the optimization problem is equivalent. The minimization of the simplified cost functional is then reduced to the minimization of certain functions that are defined on an affine space. This latter minimization is then carried out by means of the Lagrange multiplier method and explicit calculations.

The paper is organized as follows. In Section 2, we explain the two market impact models that we derive from the generalized LOB model with different resilience modes. In Section 3 , we set up the resulting optimization problem. The main results for Models 1 and 2 are presented in the respective Sections 4 and 5. In Section 6, we consider the special case of a uniform distribution of shares in the LOB as considered in [16]. In particular, we provide our new explicit formula for the optimal strategy in a block-shaped LOB as obtained in [16]. Section 7 contains numerical and theoretical studies of the optimization problem for various nonconstant shape functions. The proofs of our main results are given in the remaining Sections $\mathrm{A}$ through $\mathrm{D}$. More precisely, in Section $\mathrm{A}$ we reduce the optimization problem for our two-sided LOB models 
to the optimization over deterministic strategies within a simplified model with a collapsed bid-ask spread. The derivations of the explicit forms of the optimal strategies in Models 1 and 2 are carried out in the respective Sections $\mathrm{B}$ and $\mathrm{C}$. In Section $\mathrm{D}$ we prove the results for block-shaped LOBs from Section 6 .

\section{Two market impact models with resilience.}

In this section, we aim at modeling the dynamics of a LOB that is exposed to repeated market orders by a large trader. The overall goal of the large trader will be to purchase a large amount $X_{0}>0$ of shares within a certain time period $[0, T]$. Hence, emphasis is on buy orders, and we concentrate first on the upper part of the LOB, which consists of shares offered at various ask prices. The lowest ask price at which shares are offered is called the best ask price.

Suppose first that the large trader is not active, so that the dynamics of the limit order book are determined by the actions of noise traders only. We assume that the corresponding unaffected best ask price $A^{0}$ is a martingale on a given filtered probability space $\left(\Omega,\left(\mathcal{F}_{t}\right), \mathcal{F}, \mathbb{P}\right)$ and satisfies $A_{0}^{0}=A_{0}$. This assumption includes in particular the case in which $A^{0}$ is a Bachelier model, i.e., $A_{t}^{0}=A_{0}+\sigma W_{t}$ for an $\left(\mathcal{F}_{t}\right)$-Brownian motion $W$, as considered in [16]. We emphasize, however, that we can take any martingale and hence use, e.g., a geometric Brownian motion, which avoids the counterintuitive negative prices of the Bachelier model. Moreover, we can allow for jumps in the dynamics of $A^{0}$ so as to model the trading activities of other large traders in the market. In our context of a risk-neutral investor minimizing the expected liquidation cost, the optimal strategies will turn out to be deterministic, due to the described martingale assumption.

Above the unaffected best ask price $A_{t}^{0}$, we assume a continuous ask price distribution for available shares in the LOB: the number of shares offered at price $A_{t}^{0}+x$ is given by $f(x) d x$ for a continuous density function $f: \mathbb{R} \longrightarrow] 0, \infty[$. We will say that $f$ is the shape function of the LOB. The choice of a constant shape function corresponds to the block-shaped LOB model of Obizhaeva and Wang [16].

The shape function determines the impact of a market order placed by our large trader. Suppose for instance that the large trader places a buy market order for $x_{0}>0$ shares at time $t=0$. This market order will consume all shares located at prices between $A_{0}$ and $A_{0}+D_{0+}^{A}$, where $D_{0+}^{A}$ is determined by

$$
\int_{0}^{D_{0+}^{A}} f(x) d x=x_{0} .
$$

Consequently, the ask price will be shifted up from $A_{0}$ to

$$
A_{0+}:=A_{0}+D_{0+}^{A}
$$

see Figure 1 for an illustration.

Let us denote by $A_{t}$ the actual ask price at time $t$, i.e., the ask price after taking the price impact of previous buy orders of the large trader into account, and let us denote by

$$
D_{t}^{A}:=A_{t}-A_{t}^{0}
$$




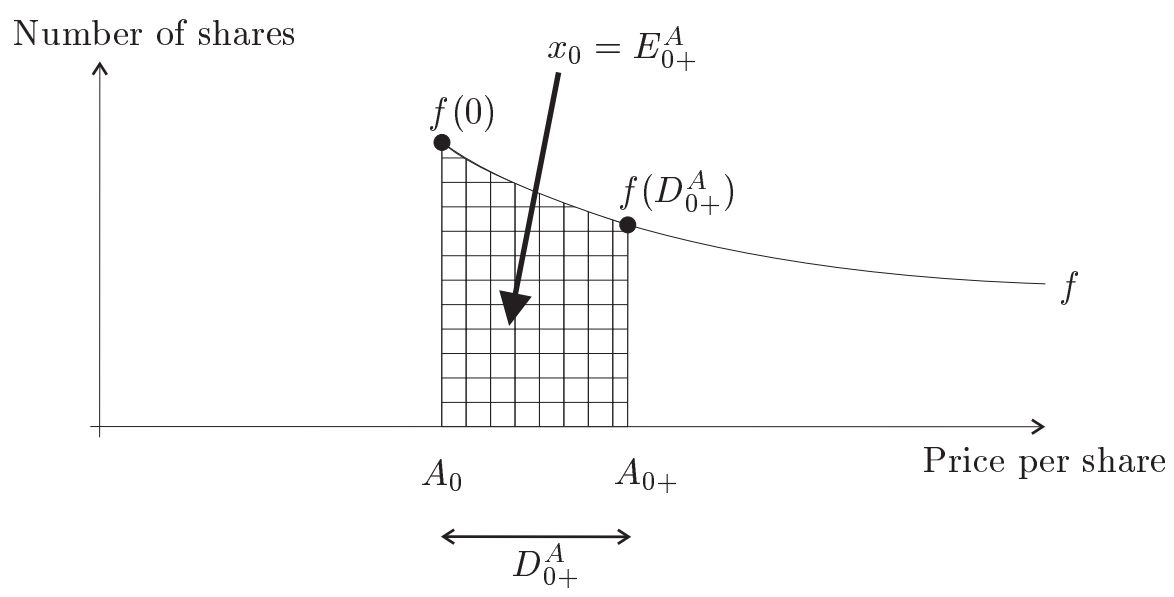

Figure 1: The impact of a buy market order of $x_{0}$ shares .

the extra spread caused by the actions of the large trader. Another buy market order of $x_{t}>0$ shares will now consume all the shares offered at prices between $A_{t}$ and

$$
A_{t+}:=A_{t}+D_{t+}^{A}-D_{t}^{A}=A_{t}^{0}+D_{t+}^{A}
$$

where $D_{t+}^{A}$ is determined by the condition

$$
\int_{D_{t}^{A}}^{D_{t+}^{A}} f(x) d x=x_{t} .
$$

Thus, the process $D^{A}$ captures the impact of market orders on the current best ask price. Clearly, the price impact $D_{t+}^{A}-D_{t}^{A}$ will be a nonlinear function of the order size $x_{t}$ unless $f$ is constant between $D_{t}^{A}$ and $D_{t+}^{A}$. Hence, our model includes the case of nonlinear impact functions; see, e.g., Almgren [2] and Almgren et al. [6] for a discussion.

Another important quantity is the process

$$
E_{t}^{A}=\int_{0}^{D_{t}^{A}} f(x) d x
$$

of the number of shares 'already eaten up' at time $t$. It quantifies the impact of the large trader on the volume of the LOB. By introducing the antiderivative

$$
F(z)=\int_{0}^{z} f(x) d x
$$

of $f$, the relation (2) can also be expressed as

$$
E_{t}^{A}=F\left(D_{t}^{A}\right) \quad \text { and } \quad D_{t}^{A}=F^{-1}\left(E_{t}^{A}\right),
$$

where we have used our assumption that $f$ is strictly positive to obtain the second identity. The relation (1) is equivalent to

$$
E_{t+}^{A}=E_{t}^{A}+x_{t}
$$


We still need to specify how $D^{A}$ and, equivalently, $E^{A}$ evolve when the large trader is inactive in between market orders. It is a well established empirical fact that order books exhibit a certain resilience as to the price impact of a large buy market orders, i.e., after the initial impact the best ask price reverts back to its previous position; cf. Biais et al. [9], Potters and Bouchaud [17], Bouchaud et al. [10], and Weber and Rosenow [21] for empirical studies. That is, at least a part of the price impact will only be temporary. For modeling this resilience, we follow Obizhaeva and Wang [16] in proposing an exponential recovery of the LOB. While in the case of a block-shaped LOB as considered in [16] the respective assumptions of exponential recovery for $D^{A}$ and for $E^{A}$ coincide, they provide two distinct possibilities for the case of a general shape function. Since either of them appears to be plausible, we will discuss them both in the sequel. More precisely, we will consider the following two models for the resilience of the market impact:

Model 1: The volume of the order book recovers exponentially, i.e., $E$ evolves according to

$$
E_{t+s}^{A}=e^{-\rho s} E_{t}^{A}
$$

if the large investor is inactive during the time interval $[t, t+s[$.

Model 2: The extra spread $D_{t}^{A}$ decays exponentially, i.e.,

$$
D_{t+s}^{A}=e^{-\rho s} D_{t}^{A}
$$

if the large investor is inactive during the time interval $[t, t+s[$.

Here the resilience speed $\rho$ is a positive constant, which for commonly traded blue chip shares will often be calibrated such that the half-life time of the exponential decay is in the order of a few minutes; see, e.g., [17, 10, 21]. Note that the dynamics of both $D^{A}$ and $E^{A}$ are now completely specified in either model.

Up to now, we have only described the effect of buy orders on the upper half of the LOB. Since the overall goal of the larger trader is to buy $X_{0}>0$ shares up to time $T$, a restriction to buy orders would seem to be reasonable. However, we do not wish to exclude the a priori possibility that, under certain market conditions, it could be beneficial to also sell some shares and to buy them back at a later point in time. To this end, we also need to model the impact of sell market orders on the lower part of the LOB, which consists of a certain number of bids for shares at each price below the best bid price. As for ask prices, we will distinguish between an unaffected best bid price, $B_{t}^{0}$, and the actual best bid price, $B_{t}$, for which the price impact of previous sell orders of the large trader is taken into account. All we assume on the dynamics of $B^{0}$ is

$$
B_{t}^{0} \leq A_{t}^{0} \quad \text { at all times } t .
$$

The distribution of bids below $B_{t}^{0}$ is modeled by the restriction of the shape function $f$ to the domain $]-\infty, 0]$. More precisely, for $x<0$, the number of bids at price $B_{t}^{0}+x$ is equal to $f(x) d x$. The quantity

$$
D_{t}^{B}:=B_{t}-B_{t}^{0},
$$

which usually will be negative, is called the extra spread in the bid price distribution. A sell market order of $x_{t}<0$ shares placed at time $t$ will consume all the shares offered at prices between $B_{t}$ and

$$
B_{t+}:=B_{t}+D_{t+}^{B}-D_{t}^{B}=B_{t}^{0}+D_{t+}^{B}
$$


where $D_{t+}^{B}$ is determined by the condition

$$
x_{t}=\int_{D_{t}^{B}}^{D_{t+}^{B}} f(x) d x=F\left(D_{t+}^{B}\right)-F\left(D_{t}^{B}\right)=E_{t+}^{B}-E_{t}^{B},
$$

for $E_{s}^{B}:=F\left(D_{s}^{B}\right)$. Note that $F$ is defined via (3) also for negative arguments. If the large trader is inactive during the time interval $\left[t, t+s\left[\right.\right.$, then the processes $D^{B}$ and $E^{B}$ behave just as their counterparts $D^{A}$ and $E^{A}$, i.e.,

$$
\begin{array}{ll}
E_{t+s}^{B}=e^{-\rho s} E_{t}^{B} & \text { in Model 1, } \\
D_{t+s}^{B}=e^{-\rho s} D_{t}^{B} & \text { in Model } 2 .
\end{array}
$$

\section{The cost minimization problem.}

When placing a single buy market order of size $x_{t} \geq 0$ at time $t$, the large trader will purchase $f(x) d x$ shares at price $A_{t}^{0}+x$, with $x$ ranging from $D_{t}^{A}$ to $D_{t+}^{A}$. Hence, the total cost of the buy market order amounts to

$$
\pi_{t}\left(x_{t}\right):=\int_{D_{t}^{A}}^{D_{t+}^{A}}\left(A_{t}^{0}+x\right) f(x) d x=A_{t}^{0} x_{t}+\int_{D_{t}^{A}}^{D_{t+}^{A}} x f(x) d x .
$$

For a sell market order $x_{t} \leq 0$, we have

$$
\pi_{t}\left(x_{t}\right):=B_{t}^{0} x_{t}+\int_{D_{t}^{B}}^{D_{t+}^{B}} x f(x) d x .
$$

In practice, very large orders are often split into a number of consecutive market orders to reduce the overall price impact. Hence, the question at hand is to determine the size of the individual orders so as to minimize a cost criterion. So let us assume that the large trader needs to buy a total of $X_{0}>0$ shares until time $T$ and that trading can occur at $N+1$ equidistant times $t_{n}=n \tau$ for $n=0, \ldots, N$ and $\tau:=T / N$. An admissible strategy will be a sequence $\xi=\left(\xi_{0}, \xi_{1}, \ldots, \xi_{N}\right)$ of random variables such that

- $\sum_{n=0}^{N} \xi_{n}=X_{0}$,

- each $\xi_{n}$ is measurable with respect to $\mathcal{F}_{t_{n}}$,

- each $\xi_{n}$ is bounded from below.

The quantity $\xi_{n}$ corresponds to the size of the market order placed at time $t_{n}$. Note that we do not a priori require $\xi_{n}$ to be positive, i.e., we also allow for intermediate sell orders, but we assume that there is some lower bound on sell orders.

The average cost $\mathcal{C}(\xi)$ of an admissible strategy $\xi$ is defined as the expected value of the total costs incurred by the consecutive market orders:

$$
\mathcal{C}(\xi)=\mathbb{E}\left[\sum_{n=0}^{N} \pi_{t_{n}}\left(\xi_{n}\right)\right]
$$


Our goal in this paper consists in finding admissible strategies that minimize the average cost within the class of all admissible strategies. For the clarity of the exposition, we decided no to treat the case of a risk averse investor. We suppose that the introduction of risk aversion will have a similar effect as in [16].

Note that the value of $\mathcal{C}(\xi)$ depends on whether we choose Model 1 or Model 2, and it will turn out that also the quantitative - though not the qualitative - features of the optimal strategies will be slightly model-dependent.

Before turning to the statements of our results, let us introduce the following standing assumption for our further analysis: the function $F$ is supposed to be unbounded in the sense that

$$
\lim _{x \uparrow \infty} F(x)=\infty \text { and } \lim _{x \downarrow-\infty} F(x)=-\infty .
$$

This assumption of unlimited order book depth is of course an idealization of reality and is for convenience only. It should not make a difference, however, as soon as the depth of the real LOB is big enough to accommodate every market order of our optimal strategy.

\section{Main theorem for Model 1.}

We will now consider the minimization of the cost functional $\mathcal{C}(\xi)$ in Model 1 , in which we assume an exponential recovery of the LOB volume; cf. (66).

Theorem 4.1 (Optimal strategy in Model 1).

Suppose that the function $h_{1}: \mathbb{R} \rightarrow \mathbb{R}_{+}$with

$$
h_{1}(y):=F^{-1}(y)-e^{-\rho \tau} F^{-1}\left(e^{-\rho \tau} y\right)
$$

is one-to-one. Then there exists a unique optimal strategy $\xi^{(1)}=\left(\xi_{0}^{(1)}, \ldots, \xi_{N}^{(1)}\right)$. The initial market order $\xi_{0}^{(1)}$ is the unique solution of the equation

$$
F^{-1}\left(X_{0}-N \xi_{0}^{(1)}\left(1-e^{-\rho \tau}\right)\right)=\frac{h_{1}\left(\xi_{0}^{(1)}\right)}{1-e^{-\rho \tau}}
$$

the intermediate orders are given by

$$
\xi_{1}^{(1)}=\cdots=\xi_{N-1}^{(1)}=\xi_{0}^{(1)}\left(1-e^{-\rho \tau}\right),
$$

and the final order is determined by

$$
\xi_{N}^{(1)}=X_{0}-\xi_{0}^{(1)}-(N-1) \xi_{0}^{(1)}\left(1-e^{-\rho \tau}\right) .
$$

In particular, the optimal strategy is deterministic. Moreover, it consists only of nontrivial buy orders, i.e., $\xi_{n}^{(1)}>0$ for all $n$.

Some remarks on this result are in order. First, the optimal strategy $\xi^{(1)}$ consists only of buy orders and so the bid price remains unaffected, i.e., we have $E_{t}^{B} \equiv 0 \equiv D_{t}^{B}$. It follows 
moreover that the process $E:=E^{A}$ is recursively given by the following Model 1 dynamics:

$$
\begin{aligned}
E_{0} & =0, \\
E_{t_{n}+} & =E_{t_{n}}+\xi_{n}^{(1)}, \quad n=0, \ldots, N, \\
E_{t_{k+1}} & =e^{-\rho \tau} E_{t_{k}+}=e^{-\rho \tau}\left(E_{t_{k}}+\xi_{k}^{(1)}\right), \quad k=0, \ldots, N-1 .
\end{aligned}
$$

Hence, by (15) and (16),

$$
E_{t_{n}+}=\xi_{0}^{(1)} \text { and } E_{t_{n+1}}=e^{-\rho \tau} \xi_{0}^{(1)} \text { for } n=0, \ldots, N-1 .
$$

That is, once $\xi_{0}^{(1)}$ has been determined via (15), the optimal strategy consists in a sequence of market orders that consume exactly that amount of shares by which the LOB has recovered since the preceding market order, due to the resilience effect. At the terminal time $t_{N}=T$, all remaining shares are bought. In the case of a block-shaped LOB, this qualitative pattern was already observed by Obizhaeva and Wang [16]. Our Theorem 4.1]now shows that this optimality pattern is actually independent of the LOB shape, thus indicating a certain robustness of optimal strategies.

Remark 4.2 According to (4) and (18), the extra spread $D:=D^{A}$ of the optimal strategy $\xi^{(1)}$ satisfies

$$
D_{t_{n}+}=F^{-1}\left(E_{t_{n}+}\right)=F^{-1}\left(\xi_{0}^{(1)}\right) .
$$

For $n=N$ we moreover have that

$$
\begin{aligned}
D_{t_{N}+} & =F^{-1}\left(E_{t_{N}+}\right)=F^{-1}\left(E_{t_{N}}+\xi_{N}^{(1)}\right) \\
& =F^{-1}\left(\xi_{0}^{(1)} e^{-\rho \tau}+X_{0}-\xi_{0}^{(1)}-(N-1) \xi_{0}^{(1)}\left(1-e^{-\rho \tau}\right)\right) \\
& =F^{-1}\left(X_{0}-N \xi_{0}^{(1)}\left(1-e^{-\rho \tau}\right)\right) .
\end{aligned}
$$

Hence, the left-hand side of (15) is equal to $D_{t_{N}+}$.

We now comment on the conditions in Theorem 4.1.

Remark 4.3 (When is $h_{1}$ one-to-one?) The function $h_{1}$ is continuous with $h_{1}(0)=0$ and $h_{1}(y)>$ 0 for $y>0$. Hence, $h_{1}$ is one-to-one if and only if $h_{1}$ is strictly increasing. We want to consider when this is the case. To this end, note that the condition

$$
h_{1}^{\prime}(y)=\frac{1}{f\left(F^{-1}(y)\right)}-\frac{e^{-2 \rho \tau}}{f\left(F^{-1}\left(e^{-\rho \tau} y\right)\right)}>0
$$

is equivalent to

$$
\ell(y):=f\left(F^{-1}\left(e^{-\rho \tau} y\right)\right)-e^{-2 \rho \tau} f\left(F^{-1}(y)\right)>0 .
$$

That is, the function $h_{1}$ will be one-to-one if, for instance, the shape function $f$ is decreasing for $y>0$ and increasing for $y<0$. In fact, it has been observed in the empirical studies [9, 17, 10, 21] that average shapes of typical order books have a maximum at or close to the best quotes and then decay as a function of the distance to the best quotes, which would conform to our assumption. 
Remark 4.4 (Continuous-time limit of the optimal strategy). One can also investigate the asymptotic behavior of the optimal strategy when the number $N$ of trades in ]0,T] tends to infinity. It is not difficult to see that $h_{1} /\left(1-e^{-\rho \tau}\right)$ converges pointwise to

$$
h_{1}^{\infty}(y):=F^{-1}(y)+\frac{y}{f\left(F^{-1}(y)\right)} .
$$

Observe also that $N\left(1-e^{-\rho \tau}\right) \rightarrow \rho T$. Since for any $N$ we have $\left.\xi_{0}^{(1)} \in\right] 0, X_{0}[$, we can extract a subsequence that converges and its limit is then necessarily solution of the equation

$$
F^{-1}\left(X_{0}-\rho T y\right)=h_{1}^{\infty}(y)
$$

If this equation has a unique solution $\xi_{0}^{(1), \infty}$ we deduce that the optimal initial trade converges to $\xi_{0}^{(1), \infty}$ when $N \longrightarrow \infty$. This is the case, for example, if $h_{1}^{\infty}$ is strictly increasing and especially when $f$ is decreasing. In that case, $N \xi_{1}^{(1)}$ converges to $\rho T \xi_{0}^{(1), \infty}$ and $\xi_{N}^{(1)}$ to $\xi_{T}^{(1), \infty}:=$ $X_{0}-\xi_{0}^{(1), \infty}(1+\rho T)$. Thus, in the continuous-time limit, the optimal strategy consists in an initial block order of $\xi_{0}^{(1), \infty}$ shares at time 0 , continuous buying at the constant rate $\rho \xi_{0}^{(1), \infty}$ during $] 0, T\left[\right.$, and a final block order of $\xi_{T}^{(1), \infty}$ shares at time $T$.

\section{Main theorem for Model 2.}

We will now consider the minimization of the cost functional

$$
\mathcal{C}(\xi)=\mathbb{E}\left[\sum_{n=0}^{N} \pi_{t_{n}}\left(\xi_{n}\right)\right]
$$

in Model 2, where we assume an exponential recovery of the extra spread; cf. (7).

Theorem 5.1 (Optimal strategy in Model 2).

Suppose that the function $h_{2}: \mathbb{R} \rightarrow \mathbb{R}$ with

$$
h_{2}(x):=x \frac{f(x)-e^{-2 \rho \tau} f\left(e^{-\rho \tau} x\right)}{f(x)-e^{-\rho \tau} f\left(e^{-\rho \tau} x\right)}
$$

is one-to-one and that the shape function satisfies

$$
\lim _{|x| \rightarrow \infty} x^{2} \inf _{z \in\left[e^{-\rho \tau} x, x\right]} f(z)=\infty .
$$

Then there exists a unique optimal strategy $\xi^{(2)}=\left(\xi_{0}^{(2)}, \ldots, \xi_{N}^{(2)}\right)$. The initial market order $\xi_{0}^{(2)}$ is the unique solution of the equation

$$
F^{-1}\left(X_{0}-N\left[\xi_{0}^{(2)}-F\left(e^{-\rho \tau} F^{-1}\left(\xi_{0}^{(2)}\right)\right)\right]\right)=h_{2}\left(F^{-1}\left(\xi_{0}^{(2)}\right)\right),
$$

the intermediate orders are given by

$$
\xi_{1}^{(2)}=\cdots=\xi_{N-1}^{(2)}=\xi_{0}^{(2)}-F\left(e^{-\rho \tau} F^{-1}\left(\xi_{0}^{(2)}\right)\right),
$$


and the final order is determined by

$$
\xi_{N}^{(2)}=X_{0}-N \xi_{0}^{(2)}+(N-1) F\left(e^{-\rho \tau} F^{-1}\left(\xi_{0}^{(2)}\right)\right) .
$$

In particular, the optimal strategy is deterministic. Moreover, it consists only of nontrivial buy orders, i.e., $\xi_{n}^{(2)}>0$ for all $n$.

Since the optimal strategy $\xi^{(2)}$ consists only of buy orders, the processes $D^{B}$ and $E^{B}$ vanish, and $D:=D^{A}$ is given by

$$
\begin{aligned}
D_{0} & =0, \\
D_{t_{n}+} & =F^{-1}\left(\xi_{n}^{(2)}+F\left(D_{t_{n}}\right)\right), \quad n=0, \ldots, N \\
D_{t_{k+1}} & =e^{-\rho \tau} D_{t_{k}+}, \quad k=0, \ldots, N-1 .
\end{aligned}
$$

Hence, induction shows that

$$
D_{t_{n}+}=F^{-1}\left(\xi_{0}^{(2)}\right) \quad \text { and } \quad D_{t_{n+1}}=e^{-\rho \tau} F^{-1}\left(\xi_{0}^{(2)}\right) \quad \text { for } n=0, \ldots, N-1 .
$$

By (41), the process $E:=E^{A}$ satisfies

$$
E_{t_{n}+}=\xi_{0}^{(2)} \quad \text { and } \quad E_{t_{n+1}}=F\left(e^{-\rho \tau} F^{-1}\left(\xi_{0}^{(2)}\right)\right) \quad \text { for } n=0, \ldots, N-1 .
$$

This is very similar to our result (18) in Model 1: once $\xi_{0}^{(1)}$ has been determined via (15), the optimal strategy consists in a sequence of market orders that consume exactly that amount of shares by which the LOB has recovered since the preceding market order. At the terminal time $t_{N}=T$, all remaining shares are bought. The only differences are in the size of the initial market order and in the mode of recovery. This qualitative similarity between the optimal strategies in Models 1 and 2 again confirms our observation of the robustness of the optimal strategy.

Remark 5.2 At the terminal time $t_{N}=T$, the extra spread is given by

$$
\begin{aligned}
D_{t_{N}+} & =F^{-1}\left(E_{t_{N}+}\right)=F^{-1}\left(E_{t_{N}}+\xi_{N}^{(2)}\right) \\
& =F^{-1}\left(X_{0}-N\left[\xi_{0}^{(2)}-F\left(e^{-\rho \tau} F^{-1}\left(\xi_{0}^{(2)}\right)\right)\right]\right),
\end{aligned}
$$

and this expression coincides with the left-hand side in (21).

Let us now comment on the conditions assumed in Theorem 5.1. To this end, we first introduce the function

$$
\widetilde{F}(z):=\int_{0}^{z} x f(x) d x
$$

Remark 5.3 If $\widetilde{F}$ is convex then condition (20) in Theorem 5.1 is satisfied. This fact admits the following short proof. Take $x^{*} \in\left[e^{-\rho \tau} x, x\right]$ realizing the infimum of $f$ in $\left[e^{-\rho \tau} x, x\right]$. Then

$$
x^{2} \inf _{z \in\left[e^{-\rho \tau} x, x\right]} f(z)=x^{2} f\left(x^{*}\right) \geq x^{*}\left(x^{*} f\left(x^{*}\right)\right) .
$$

Due to the convexity of $\widetilde{F}$, its derivative $\widetilde{F}^{\prime}(x)=x f(x)$ is increasing. It is also nonzero iff $x \neq 0$. Therefore the right-hand side of (25) tends to infinity for $|x| \rightarrow \infty$. 
However, the convexity of $\widetilde{F}$ is not necessary for condition (20) as is illustrated by the following simple example.

Example 5.4 Let us construct a shape function for which (20) is satisfied even though $\widetilde{F}$ need not be convex. To this end, take any continuous function $b: \mathbb{R} \rightarrow] 0, \infty[$ that is bounded away from zero. Then let

$$
f(x):= \begin{cases}b(1) & |x| \leq 1 \\ \frac{b(x)}{\sqrt{|x|}} & |x|>1 .\end{cases}
$$

This shape function clearly satisfies condition (20). Taking for example $b(x)=1+\varepsilon \cos (x)$ with $0<\varepsilon<1$, however, gives a nonconvex function $\widetilde{F}$. Moreover, by choosing $\varepsilon$ small enough, we can obtain $h_{2}^{\prime}(x)>0$ so that the shape function $f$ satisfies the assumptions of Theorem 5.1 .

We now comment on the condition that $h_{2}$ is one-to-one. The following example shows that this is indeed a nontrivial assumption.

Example 5.5 We now provide an example of a shape function $f$ for which the corresponding function $h_{2}$ is not one-to-one. First note that $h_{2}(0)=0$ and

$$
\lim _{\epsilon \downarrow 0} \frac{h_{2}(\epsilon)-h_{2}(0)}{\epsilon}=\frac{1-e^{-2 \rho \tau}}{1-e^{-\rho \tau}}>0 .
$$

Therefore and since $h_{2}$ is continuous, it cannot be one-to-one if we can find $x^{*}>0$ such that $h_{2}\left(x^{*}\right)<0$. To this end, we assume that there exist $n \in\{2,3, \ldots\}$ such that $e^{-\rho \tau}=\frac{1}{n}$ and take

$$
f(x):= \begin{cases}(n+1) & x \in\left[0, \frac{1}{n}\right) \\ (n+1)-\frac{n^{2}}{n-1}\left(x-\frac{1}{n}\right) & x \in\left[\frac{1}{n}, 1\right] \\ 1 & x \in(1, \infty) ;\end{cases}
$$

see Figure 2. Furthermore, we define $x^{*}:=1$ to obtain

$$
h_{2}\left(x^{*}\right)=\frac{n^{2}-(n+1)}{-n}<0 .
$$

The intuition why Theorem 4.1 can be applied to this LOB shape (f is decreasing), but Theorem 5.1 cannot be used, is the following: For the first trade $\xi_{0}^{(2)}$ from (21) we might get $D_{t_{n+1}}=e^{-\rho \tau} F^{-1}\left(\xi_{0}^{(2)}\right) \geq 1$, i.e. there are only few new shares from the resilience effect since $f(x)$ is low for $x \geq 1$. But this $\xi_{0}^{(2)}$ would not be optimal1 1 . We cannot have this phenomenon in Model 1 because there the resilience is proportional to the volume consumed by the large investor.

\footnotetext{
${ }^{1}$ Take e.g. $n=2$ and $e^{-\rho \tau}=1 / 2$. Then for $X_{0}=N+\frac{9}{2}$ we get from (21) $\xi_{0}^{(2)}=\frac{7}{2}, D_{t_{n+1}}=1$ and $\xi_{1}^{(2)}=\ldots=\xi_{N-1}^{(2)}=1, \xi_{N}^{(2)}=2$. The corresponding cost are higher than for the alternative strategy $\bar{\xi}_{0}^{(2)}=\frac{5}{2}$, $\bar{\xi}_{1}^{(2)}=\ldots=\bar{\xi}_{N-1}^{(2)}=1, \bar{\xi}_{N}^{(2)}=3$.
} 


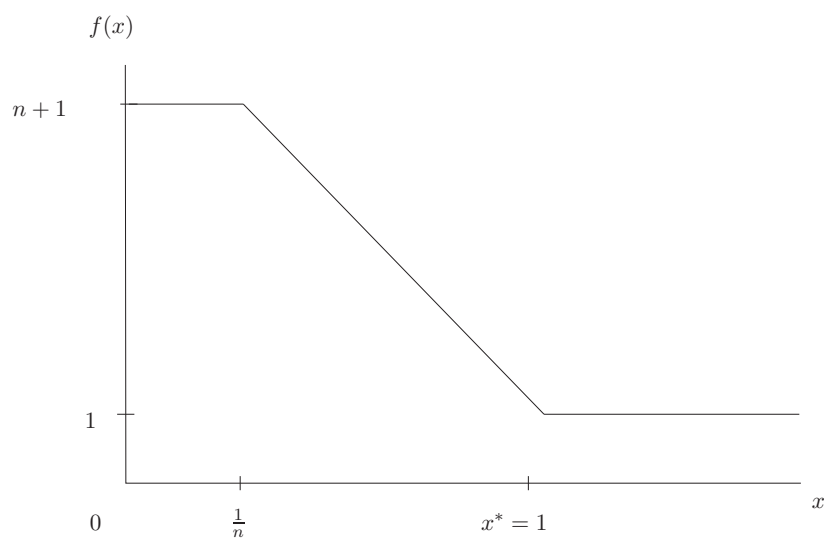

Figure 2: A shape function $f$ for which the function $h_{2}$ is not one-to-one.

Remark 5.6 (Continuous-time limit of the optimal strategy). As in Remark 4.4. we can study the asymptotic behavior of the optimal strategy as the number $N$ of trades in ]0,T[ tends to infinity. First, we can check that $h_{2}$ converges pointwise to

$$
h_{2}^{\infty}(x):=x\left(1+\frac{f(x)}{f(x)+x f^{\prime}(x)}\right),
$$

and that $N\left(y-F\left(e^{-\rho \tau} F^{-1}(y)\right)\right)$ tends to $\rho T F^{-1}(y) f\left(F^{-1}(y)\right)$, provided that $f$ is continuously differentiable. Now, suppose that the equation

$$
F^{-1}\left(X_{0}-\rho T F^{-1}(y) f\left(F^{-1}(y)\right)\right)=h_{2}^{\infty}\left(F^{-1}(y)\right)
$$

has a unique solution on $] 0, X_{0}\left[\right.$, which we will call $\xi_{0}^{(2), \infty}$. We can check that $\xi_{0}^{(2), \infty}$ is the only one possible limit for a subsequence of $\xi_{0}^{(2)}$, and it is therefore its limit. We can then show that $N \xi_{1}^{(2)}$ converges to $\rho T F^{-1}\left(\xi_{0}^{(2), \infty}\right) f\left(F^{-1}\left(\xi_{0}^{(2), \infty}\right)\right)$ and $\xi_{N}^{(2)}$ to

$$
\xi_{T}^{(2), \infty}:=X_{0}-\xi_{0}^{(2), \infty}-\rho T F^{-1}\left(\xi_{0}^{(2), \infty}\right) f\left(F^{-1}\left(\xi_{0}^{(2), \infty}\right)\right) .
$$

Thus, in the continuous-time limit, the optimal strategy consists in an initial block order of $\xi_{0}^{(2), \infty}$ shares at time 0 , continuous buying at the constant rate $\rho F^{-1}\left(\xi_{0}^{(2), \infty}\right) f\left(F^{-1}\left(\xi_{0}^{(2), \infty}\right)\right)$ during ]0,T[, and a final block order of $\xi_{T}^{(2), \infty}$ shares at time $T$.

\section{Closed form solution for block-shaped LOBs and ad- ditional permanent impact.}

In this first example section, we consider a block-shaped LOB corresponding to a constant shape function $f(x) \equiv q$ for some $q>0$. In this case, there is no difference between Models 1 and 2. Apart from our more general dynamics for $A^{0}$, the main difference to the market impact model introduced by Obizhaeva and Wang [16] is that, for the moment, we do not consider a permanent impact of market orders. In Corollary 6.4, we will see, however, that our results yield a closed-form solution even in the case of nonvanishing permanent impact.

By applying either Theorem 4.1 or Theorem 5.1 we obtain the following Corollary. 
Corollary 6.1 (Closed-form solution for block-shaped LOB).

In a block-shaped $L O B$, the unique optimal strategy $\xi^{*}$ is

$$
\xi_{0}^{*}=\xi_{N}^{*}=\frac{X_{0}}{(N-1)\left(1-e^{-\rho \tau}\right)+2} \quad \text { and } \quad \xi_{1}^{*}=\cdots=\xi_{N-1}^{*}=\frac{X_{0}-2 \xi_{0}^{*}}{N-1} .
$$

The preceding result extends [16, Proposition 1] in several aspects. First, we do not focus on the Bachelier model but admit arbitrary martingale dynamics for our unaffected best ask price $A^{0}$. Second, only static, deterministic buy order strategies are considered in [16], while we here allow our admissible strategies to be adapted and to include sell orders. Since, a posteriori, our optimal strategy turns out to be deterministic and positive, it is clear that it must coincide with the optimal strategy from [16, Proposition 1]. Our strategy (27) therefore also provides an explicit closed-form solution of the recursive scheme obtained in [16]. We recall this recursive scheme in (31) below.

On the other hand, Obizhaewa and Wang [16] allow for an additional permanent impact of market orders. Intuitively, in a block-shaped LOB with $f \equiv q>0$, the permanent impact of a market order $x_{t}$ means that only a certain part of the impact of $x_{t}$ decays to zero, while the remaining part remains forever present in the LOB. More precisely, the impact of an admissible buy order strategy $\xi$ on the extra spread $D^{A}$ is given by the dynamics

$$
D_{t}^{A}=\lambda \sum_{t_{k}<t} \xi_{k}+\sum_{t_{k}<t} \kappa e^{-\rho\left(t-t_{k}\right)} \xi_{k}
$$

where $\lambda<1 / q$ is a constant quantifying the permanent impact and

$$
\kappa:=\frac{1}{q}-\lambda
$$

is the proportion of the temporary impact. Note that, for $\lambda=0$, we get back our dynamics (6) and (7), due to the fact that we consider a block-shaped LOB. It will be convenient to introduce the process $X_{t}$ of the still outstanding number of shares at time $t$ when using an admissible strategy:

$$
X_{t}:=X_{0}-\sum_{t_{k}<t} \xi_{k}
$$

We can now state the result by Obizhaeva and Wang.

Proposition 6.2 [16, Proposition 1] In a block-shaped LOB with permanent impact $\lambda$, the optimal strategy $\xi^{O W}$ in the class of deterministic strategies is determined by the forward scheme

$$
\begin{aligned}
& \xi_{n}^{O W}=\frac{1}{2} \delta_{n+1}\left[\epsilon_{n+1} X_{t_{n}}-\phi_{n+1} D_{t_{n}}\right], \quad n=0, \ldots, N-1, \\
& \xi_{N}^{O W}=X_{T},
\end{aligned}
$$

where $\delta_{n}, \epsilon_{n}$ and $\phi_{n}$ are defined by the backward scheme

$$
\begin{aligned}
\delta_{n} & :=\left(\frac{1}{2 q}+\alpha_{n}-\beta_{n} \kappa e^{-\rho \tau}+\gamma_{n} \kappa^{2} e^{-2 \rho \tau}\right)^{-1} \\
\epsilon_{n} & :=\lambda+2 \alpha_{n}-\beta_{n} \kappa e^{-\rho \tau} \\
\phi_{n} & :=1-\beta_{n} e^{-\rho \tau}+2 \gamma_{n} \kappa e^{-2 \rho \tau} .
\end{aligned}
$$


with $\alpha_{n}, \beta_{n}$ and $\gamma_{n}$ given by

$$
\begin{aligned}
\alpha_{N}=\frac{1}{2 q}-\lambda \text { and } \alpha_{n} & =\alpha_{n+1}-\frac{1}{4} \delta_{n+1} \epsilon_{n+1}^{2}, \\
\beta_{N}=1 \text { and } \beta_{n} & =\beta_{n+1} e^{-\rho \tau}+\frac{1}{2} \delta_{n+1} \epsilon_{n+1} \phi_{n+1}, \\
\gamma_{N}=0 & \text { and } \gamma_{n}=\gamma_{n+1} e^{-2 \rho \tau}-\frac{1}{4} \delta_{n+1} \phi_{n+1}^{2} .
\end{aligned}
$$

It is a priori clear that for $\lambda=0$ the explicit optimal strategy obtained in Corollary 6.1 must coincide with the strategy $\xi^{O W}$ obtained via the recursive scheme (31) in Proposition 6.2. To cross-check our results with the ones in [16], we will nevertheless provide an explicit and independent proof of the following proposition. It can be found in Section D.

Proposition 6.3 For $\lambda=0$, the optimal strategy (27) of Corollary 6.1 solves the recursive scheme (31) in Proposition 6.2.

Let us now extend our results so as to obtain the explicit solution of (31) even with nonvanishing permanent impact. To this end, we note that the optimal strategy $\xi^{O W}=\left(\xi_{0}^{O W}, \ldots, \xi_{N}^{O W}\right)$ is obtained in [16] as the unique minimizer of the cost functional

$$
C_{\lambda, q}^{\mathrm{OW}}: \mathbb{R}^{N+1} \rightarrow \mathbb{R}
$$

defined by

$$
\begin{aligned}
& C_{\lambda, q}^{\mathrm{OW}}\left(x_{0}, \ldots, x_{N}\right) \\
& =A_{0} \sum_{i=0}^{N} x_{i}+\frac{\lambda}{2}\left(\sum_{i=0}^{N} x_{i}\right)^{2}+\kappa \sum_{k=0}^{N}\left(\sum_{i=0}^{k-1} x_{i} e^{-\rho(k-i) \tau}\right) x_{k}+\frac{\kappa}{2} \sum_{i=0}^{N} x_{i}^{2},
\end{aligned}
$$

where $\kappa$ is as in (29). Now we just have to observe that

$$
C_{\lambda, q}^{\mathrm{OW}}\left(x_{0}, \ldots, x_{N}\right)=\frac{\lambda}{2}\left(\sum_{i=0}^{N} x_{i}\right)^{2}+C_{0, \kappa^{-1}}^{\mathrm{OW}}\left(x_{0}, \ldots, x_{N}\right) .
$$

Therefore, under the constraint $\sum_{i=0}^{N} x_{i}=X_{0}$, it is equivalent to minimize either $C_{\lambda, q}^{\mathrm{OW}}$ or $C_{0, \kappa^{-1}}^{\mathrm{OW}}$. We already know that the optimal strategy $\xi^{*}$ of Corollary 6.1 minimizes $C_{0, q}^{\text {OW }}$. But $\xi^{*}$ is in fact independent of $q$. Hence, $\xi^{*}$ also minimizes $C_{0, \kappa^{-1}}^{\mathrm{OW}}$ and in turn $C_{\lambda, q}^{\mathrm{OW}}$. We have therefore proved:

Corollary 6.4 The optimal strategy $\xi^{*}$ of Corollary 6.1 is the unique optimal strategy in any block-shaped $L O B$ with permanent impact $\lambda<1 / q$. In particular, it solves the recursive scheme (31).

The last part of the assertion of Corollary 6.4 is remarkable insofar as the recursive scheme (31) depends on both $q$ and $\lambda$ whereas the optimal strategy $\xi^{*}$ does not. 


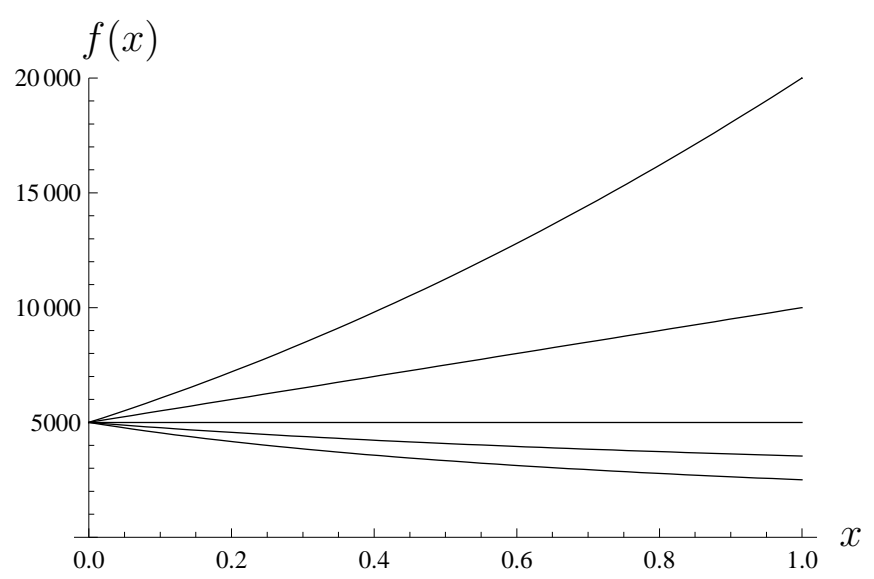

Figure 3: Plots of the power law shape functions for $q=5,000$ shares and exponent $\alpha=$ $-2,-1,0, \frac{1}{2}$ and 1 top down. Please note that these examples do not necessarily correspond to real-world shape functions.

\section{Examples.}

In this section, we consider the power law family $f: \mathbb{R} \rightarrow \mathbb{R}_{>0}$ with

$$
f(x)=\frac{q}{(|x|+1)^{\alpha}}
$$

as example shape functions. The antiderivative of the shape function and its inverse are

$$
F(x)=\left\{\begin{array}{ll}
q \log (x+1) & \text { if } \alpha=1 \\
q x & \text { if } \alpha=0 \\
\frac{q}{1-\alpha}\left[(x+1)^{1-\alpha}-1\right] & \text { otherwise }
\end{array} \quad F^{-1}(y)= \begin{cases}e^{\frac{y}{q}}-1 & \text { if } \alpha=1 \\
\frac{y}{q} & \text { if } \alpha=0 \\
{\left[1+(1-\alpha) \frac{y}{q}\right]^{\frac{1}{1-\alpha}}-1} & \text { otherwise }\end{cases}\right.
$$

for positive values of $x$ and $y$. Set $F(x)=-F(-|x|)$ and $F^{-1}(y)=-F^{-1}(-|y|)$ for $x, y<0$.

One can easily check that the assumptions of both Theorem 4.1 and Theorem 5.1 are satisfied for $\alpha \leq 1$. It is remarkable that the optimal strategies (Figure 4) vary only slightly when changing $\alpha$ or the resilience mode. This observation provides further evidence for the robustness and stability of the optimal strategy, and this time not only on a qualitative but also on a quantitative level.

From Figure 4 one recognizes some monotonicity properties of the optimal strategies. We want to give some intutition to understand these. Let us start with Model 1 . There the dynamics of $E_{t}$ do not depend on the LOB shape, but solely on the strategy. Only the cost depends on $f$. We know from the constant LOB case that the optimum strategy is not sensible to the value of $f\left(\xi_{0}^{(1)}\right)$. This explains why there are few quantitative differences for Model 1 along the different LOB shapes. Moreover, $\xi_{1}^{(1)}=(1-a) \xi_{0}^{(1)}$ with $a:=e^{-\rho \tau}$ is proportional to $\xi_{0}^{(1)}$ since it is the number of shares that reappear between two trades. Therefore the optimal strategy is just a trade-off between $\xi_{0}^{(1)}$ and $\xi_{N}^{(1)}$. When $f$ is increasing (decreasing), the first trade is relatively more (less) expansive compared to the last one. This explains that $\xi_{0}^{(1)}<\xi_{N}^{(1)}$ for $\alpha<0$ and $\xi_{0}^{(1)}>\xi_{N}^{(1)}$ for $\alpha>0$. With 'relatively' we mean 'with respect to the constant LOB case' $(\alpha=0)$ where $\xi_{0}=\xi_{N}$. 

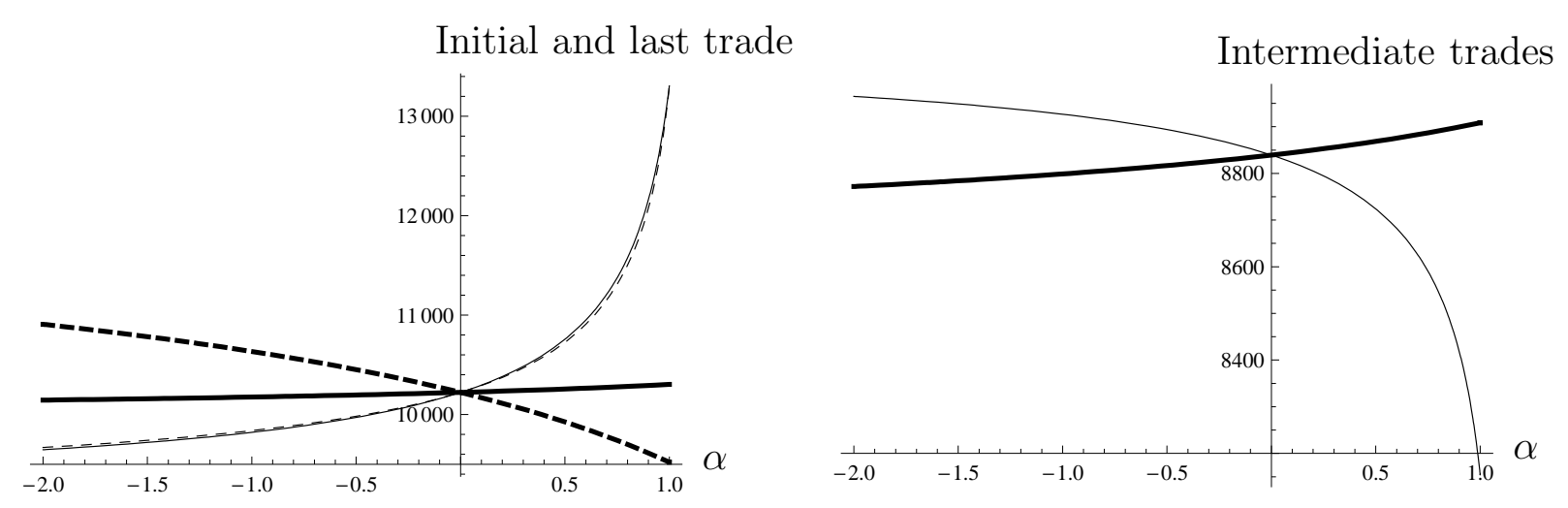

Figure 4: The plots show the optimal strategies for varying exponents $\alpha$. We set $X_{0}=100,000$ and $q=5,000$ shares, $\rho=20, T=1$ and $N=10$. In the left figure we see $\xi_{0}^{(1)}$ (dashed and thick), $\xi_{N}^{(1)}$ (thick line) and $\xi_{0}^{(2)}, \xi_{N}^{(2)}$. The figure on the right hand side shows $\xi_{1}^{(1)}$ (thick line) and $\xi_{1}^{(2)}$.

For Model 2 the dynamics of $E_{t}$ do depend on the shape function, which explains more substantial variations according to $f$. Here the main idea is to realize that, for increasing (decreasing) shape functions, resilience of the volume is stronger (weaker) in comparison to Model 1. Indeed, we have then $x-F\left(a F^{-1}(x)\right) \geq x(1-a)$ (resp. $\left.x-F\left(a F^{-1}(x)\right) \leq x(1-a)\right)$. Therefore $\xi_{1}^{(1)}<\xi_{1}^{(2)}\left(\xi_{1}^{(1)}>\xi_{1}^{(2)}\right)$ and the discrete trades $\xi_{0}^{(2)}$ and $\xi_{N}^{(2)}$ are lower (higher) as in Model 1. These effects are the more pronounced the steeper the LOB shape. Furthermore, there is the tendency that $\xi_{0}^{(2)} \approx \xi_{N}^{(2)}$. On the one hand, the same argument as in Model 1 suggests $\xi_{0}^{(2)}<\xi_{N}^{(2)}$ for increasing $f$. But on the other hand, for an increasing shape function the number of reappearing shares grows disproportionately in the initial trade which favors the initial trade being higher than the last trade. These two effects seem to counterbalance each other.

Remark 7.1 Taking the special LOB shape $f(x)=\frac{q}{\sqrt{1+\mu|x|}}, q>0$ and $\mu \geq 0$ we can solve explicitly the optimal strategy in Model 1 from Theorem 4.1. The optimal initial trade is given by

$$
\begin{aligned}
\xi_{0}^{(1)}= & \frac{1+a+N(1-a)\left(1+(\mu / 2 q) X_{0}\right)}{(\mu / 2 q)\left(N^{2}(1-a)^{2}-\left(1+a+a^{2}\right)\right)} \\
& -\frac{\sqrt{(N+1-a(N-1))^{2}+(\mu / q) X_{0}\left[N\left(1-a^{2}\right)+\left(1+a+a^{2}\right)\left(1+(\mu / 4 q) X_{0}\right)\right]}}{(\mu / 2 q)\left(N^{2}(1-a)^{2}-\left(1+a+a^{2}\right)\right)},
\end{aligned}
$$

and we can show that it is increasing with respect to the parameter $\mu$ that tunes the slope of the $L O B$.

\section{A Reduction to the case of deterministic strategies.}

In this section, we prepare for the proofs of Theorems 4.1 and 5.1 by reducing the minimization of the cost functional

$$
\mathcal{C}(\xi)=\mathbb{E}\left[\sum_{n=0}^{N} \pi_{t_{n}}\left(\xi_{n}\right)\right]
$$


with respect to all admissible strategies $\xi$ to the minimization of certain cost functions $C^{(i)}$ : $\mathbb{R}^{N+1} \rightarrow \mathbb{R}$, where $i=1,2$ refers to the model under consideration.

To this end, we introduce simplified versions of the model dynamics by collapsing the bidask spread into a single value. More precisely, for any admissible strategy $\xi$, we introduce a new pair of processes $D$ and $E$ that react on both sell and buy orders according to the following dynamics.

- We have $E_{0}=D_{0}=0$ and

$$
E_{t}=F\left(D_{t}\right) \quad \text { and } \quad D_{t}=F^{-1}\left(E_{t}\right) .
$$

- For $n=0, \ldots, N$, regardless of the sign of $\xi_{n}$,

$$
E_{t_{n}+}=E_{t_{n}}+\xi_{n} \quad \text { and } \quad D_{t_{n}+}=F^{-1}\left(\xi_{n}+F\left(D_{t_{n}}\right)\right) .
$$

- For $k=0, \ldots, N-1$,

$$
\begin{array}{ll}
E_{t_{k+1}}=e^{-\rho \tau} E_{t_{k}+} & \text { in Model 1, } \\
D_{t_{k+1}}=e^{-\rho \tau} D_{t_{k}+} & \text { in Model } 2 .
\end{array}
$$

The values of $E_{t}$ and $D_{t}$ for $t \notin\left\{t_{0}, \ldots, t_{N}\right\}$ will not be needed in the sequel. Note that $E=E^{A}$ and $D=D^{A}$ if $\xi$ consists only of buy orders, while $E=E^{B}$ and $D=D^{B}$ if $\xi$ consists only of sell orders. In general, we will only have

$$
E_{t}^{B} \leq E_{t} \leq E_{t}^{A} \quad \text { and } \quad D_{t}^{B} \leq D_{t} \leq D_{t}^{A} .
$$

We now introduce the simplified price of $\xi_{n}$ at time $t_{n}$ by

$$
\bar{\pi}_{t_{n}}\left(\xi_{n}\right):=A_{t_{n}}^{0} \xi_{n}+\int_{D_{t_{n}}}^{D_{t_{n}+}} x f(x) d x,
$$

regardless of the sign of $\xi_{n}$. Using (38) and (18), we easily get

$$
\bar{\pi}_{t_{n}}\left(\xi_{n}\right) \leq \pi_{t_{n}}\left(\xi_{n}\right) \quad \text { with equality if } \xi_{k} \geq 0 \text { for all } k \leq n \text {. }
$$

The simplified price functional is defined as

$$
\overline{\mathcal{C}}(\xi):=\mathbb{E}\left[\sum_{n=0}^{N} \bar{\pi}_{t_{n}}\left(\xi_{n}\right)\right] .
$$

We will show that, in Model $i \in\{1,2\}$, the simplified price functional $\overline{\mathcal{C}}$ has a unique minimizer, which coincides with the corresponding optimal strategy $\xi^{(i)}$ as described in the respective theorem. We will also show that $\xi^{(i)}$ consists only of buy orders, so that (40) will yield $\mathcal{C}\left(\xi^{(i)}\right)=$ $\overline{\mathcal{C}}\left(\xi^{(i)}\right)$. Consequently, $\xi^{(i)}$ must be the unique minimizer of $\mathcal{C}$.

Let us now reduce the minimization of $\overline{\mathcal{C}}$ to the minimization of functionals $C^{(i)}$ defined on deterministic strategies. To this end, let us use the notation

$$
X_{t}:=X_{0}-\sum_{t_{k}<t} \xi_{k} \text { for } t \leq T \text { and } X_{t_{N+1}}:=0 .
$$


The accumulated simplified price of an admissible strategy $\xi$ is

$$
\sum_{n=0}^{N} \bar{\pi}_{t_{n}}\left(\xi_{n}\right)=\sum_{n=0}^{N} A_{t_{n}}^{0} \xi_{n}+\sum_{n=0}^{N} \int_{D_{t_{n}}}^{D_{t_{n}+}} x f(x) d x .
$$

Integrating by parts yields

$$
\sum_{n=0}^{N} A_{t_{n}}^{0} \xi_{n}=-\sum_{n=0}^{N} A_{t_{n}}^{0}\left(X_{t_{n+1}}-X_{t_{n}}\right)=X_{0} A_{0}+\sum_{n=1}^{N} X_{t_{n}}\left(A_{t_{n}}^{0}-A_{t_{n-1}}^{0}\right) .
$$

Since $\xi$ is admissible, $X_{t}$ is a bounded predictable process. Hence, due to the martingale property of the unaffected best ask process $A^{0}$, the expectation of (42) is equal to $X_{0} A_{0}$.

Next, observe that, in each Model $i=1,2$, the simplified extra spread process $D$ evolves deterministically once the values $\xi_{0}, \xi_{1}(\omega), \ldots, \xi_{N}(\omega)$ are given. Hence, there exists a deterministic function $C^{(i)}: \mathbb{R}^{N+1} \rightarrow \mathbb{R}$ such that

$$
\sum_{n=0}^{N} \int_{D_{t_{n}}}^{D_{t_{n}+}} x f(x) d x=C^{(i)}\left(\xi_{0}, \ldots, \xi_{N}\right)
$$

It follows that

$$
\overline{\mathcal{C}}(\xi)=A_{0} X_{0}+\mathbb{E}\left[C^{(i)}\left(\xi_{0}, \ldots, \xi_{N}\right)\right] .
$$

We will show in the respective Sections $\left[\mathrm{B}\right.$ and $\mathrm{C}$ that the functions $C^{(i)}, i=1,2$, have unique minima within the set

$$
\Xi:=\left\{\left(x_{0}, \ldots, x_{N}\right) \in \mathbb{R}^{N+1} \mid \sum_{n=0}^{N} x_{n}=X_{0}\right\}
$$

and that these minima coincide with the values of the optimal strategies $\xi^{(i)}$ as provided in Theorems 4.1 and 5.1. This concludes the reduction to the case of deterministic strategies. We will now turn to the minimization of the functions $C^{(i)}$ over $\Xi$. To simplify the exposition, let us introduce the following shorthand notation in the sequel:

$$
a:=e^{-\rho \tau} .
$$

\section{B The optimal strategy in Model 1.}

In this section, we will minimize the function $C^{(1)}$ of (43) over the set $\Xi$ of all deterministic strategies and thereby complete the proof of Theorem 4.1. To this end, recall first the definition of the two processes $E$ and $D$ as given in (35)-(37). Based on their Model 1 dynamics, we will now obtain a formula of the cost function $C^{(1)}$ of (43) in terms of the functions $F$ and $\widetilde{F}$. It will be convenient to introduce also the function

$$
G(y):=\widetilde{F}\left(F^{-1}(y)\right) .
$$


Then we have for any deterministic strategy $\xi=\left(x_{0}, \ldots, x_{N}\right) \in \Xi$ that

$$
\begin{aligned}
C^{(1)}\left(x_{0}, \ldots, x_{N}\right)= & \sum_{n=0}^{N} \int_{D_{t_{n}}}^{D_{t_{n}}+} x f(x) d x \\
= & \sum_{n=0}^{N}\left(\widetilde{F}\left(F^{-1}\left(E_{t_{n}}\right)\right)-\widetilde{F}\left(F^{-1}\left(E_{t_{n}}\right)\right)\right) \\
= & \sum_{n=0}^{N}\left(G\left(E_{t_{n}}+x_{n}\right)-G\left(E_{t_{n}}\right)\right) \\
= & G\left(x_{0}\right)-G(0) \\
& +G\left(a x_{0}+x_{1}\right)-G\left(a x_{0}\right) \\
& +G\left(a^{2} x_{0}+a x_{1}+x_{2}\right)-G\left(a^{2} x_{0}+a x_{1}\right) \\
& +\ldots \\
& +G\left(a^{N} x_{0}+\cdots+x_{N}\right)-G\left(a^{N} x_{0}+\cdots+a x_{N-1}\right) .
\end{aligned}
$$

The derivative of $G$ is

$$
G^{\prime}(y)=\widetilde{F}^{\prime}\left(F^{-1}(y)\right)\left(F^{-1}\right)^{\prime}(y)=F^{-1}(y) f\left(F^{-1}(y)\right) \frac{1}{f\left(F^{-1}(y)\right)}=F^{-1}(y) .
$$

Hence, $G$ is twice continuously differentiable, positive and convex. The cost function $C^{(1)}$ is also twice continuously differentiable.

Lemma B.1 We have $C^{(1)}\left(x_{0}, \ldots, x_{N}\right) \longrightarrow+\infty$ for $|\xi| \rightarrow \infty$, and therefore there exists a local minimum of $C^{(1)}$ in $\Xi$.

Proof: Using (48) and the fact that $F^{-1}(y x)$ is increasing, we get that for all $y \in \mathbb{R}$ and $c \in(0,1]$

$$
G(y)-G(c y) \geq(1-c) \cdot\left|F^{-1}(c y)\right| \cdot|y| .
$$

Let us rearrange the sum in (47) in order to use inequality (49). We obtain

$$
\begin{aligned}
& C^{(1)}\left(x_{0}, \ldots, x_{N}\right) \\
&= G\left(a^{N} x_{0}+a^{N-1} x_{1}+\cdots+x_{N}\right)-G(0) \\
&+\sum_{n=0}^{N-1}\left[G\left(a^{n} x_{0}+\cdots+x_{n}\right)-G\left(a\left(a^{n} x_{0}+\cdots+x_{n}\right)\right)\right] \\
& \geq G\left(a^{N} x_{0}+a^{N-1} x_{1}+\cdots+x_{N}\right)-G(0) \\
& \quad(1-a) \sum_{n=0}^{N-1}\left|F^{-1}\left(a\left(a^{n} x_{0}+\cdots+x_{n}\right)\right)\right|\left|a^{n} x_{0}+\cdots+x_{n}\right| .
\end{aligned}
$$

Let us denote by $T_{1}: \mathbb{R}^{N+1} \rightarrow \mathbb{R}^{N+1}$ the linear mapping

$$
T_{1}\left(x_{0}, \ldots, x_{n}\right)=\left(x_{0}, a x_{0}+x_{1}, \ldots, a^{N} x_{0}+x_{1} a^{N-1}+\cdots+x_{N}\right) .
$$


It is non trivial and therefore the norm of $T_{1}\left(x_{0}, \ldots, x_{N}\right)$ tends to infinity as the norm of its argument goes to infinity. Because $F$ is unbounded, we know that both $G(y)$ and $\left|F^{-1}(a y)\right||y|$ tend to infinity for $|y| \rightarrow \infty$. Let us introduce

$$
H(y)=\min \left(G(y),\left|F^{-1}(a y)\right||y|\right) .
$$

Then also $H(y) \longrightarrow+\infty$ for $|y| \rightarrow \infty$, and we conclude that

$$
C^{(1)}\left(x_{0}, \ldots, x_{N}\right) \geq(1-a) H\left(\left|T_{1}\left(x_{0}, \ldots, x_{N}\right)\right|_{\infty}\right)-G(0),
$$

where $|\cdot|_{\infty}$ denotes the $\ell^{\infty}$-norm on $\mathbb{R}^{N+1}$. Hence, the assertion follows.

We now consider Equation (15) in Theorem 4.1, which we recall here for the convenience of the reader:

$$
F^{-1}\left(X_{0}-N x_{0}(1-a)\right)=\frac{h_{1}\left(x_{0}\right)}{1-a} .
$$

This equation is solved by $x_{0}$ if and only if $x_{0}$ is a zero of the function

$$
\hat{h}_{1}(y):=h_{1}(y)-(1-a) F^{-1}\left(X_{0}-N y(1-a)\right) .
$$

Lemma B.2 Under the assumptions of the Theorem 4.1, $\hat{h}_{1}$ has at most one zero $x_{0}$, which, if it exists, is necessarily positive.

Proof: It is sufficient to show that $\hat{h}_{1}$ is strictly increasing. We know that $h_{1}(0)=0, h_{1}(y)>0$ for $y>0$, and $h_{1}$ is continuous and one-to-one. Consequently, $h_{1}$ must be strictly increasing and therefore

$$
\hat{h}_{1}^{\prime}(y)=h_{1}^{\prime}(y)+\frac{N(a-1)^{2}}{f\left(F^{-1}\left(X_{0}+N y(a-1)\right)\right)}>0 .
$$

Furthermore, if there exists a solution $x_{0}$, then it must be positive since

$$
\hat{h}_{1}(0)=(a-1) F^{-1}\left(X_{0}\right)<0 .
$$

Theorem 4.1 will now follow by combining the following proposition with the arguments explained in Section A.

Proposition B.3 The function $C^{(1)}: \Xi \rightarrow \mathbb{R}$ has the strategy $\xi^{(1)}$ from Theorem 4.1 as its unique minimizer. Moreover, the components of $\xi^{(1)}$ are all strictly positive. 
Proof: Thanks to Lemma B.1, there is at least one optimal strategy $\xi^{*}=\left(x_{0}^{*}, \ldots, x_{N}^{*}\right) \in \Xi$, and standard results give the existence of a Lagrange multiplier $\nu \in \mathbb{R}$ such that

$$
\frac{\partial}{\partial x_{i}} C^{(1)}\left(x_{0}^{*}, \ldots, x_{N}^{*}\right)=\nu \quad \text { for } i=0, \ldots, N
$$

Now we use the form of $C^{(1)}$ as given in (47) to obtain the following relation between the partial derivatives of $C^{(1)}$ for $i=0, \ldots, N-1$ :

$$
\begin{aligned}
\frac{\partial}{\partial x_{i}} C^{(1)}\left(x_{0}, \ldots, x_{N}\right) & =a\left[\frac{\partial}{\partial x_{i+1}} C^{(1)}\left(x_{0}, \ldots, x_{N}\right)-G^{\prime}\left(a\left(a^{i} x_{0}+\cdots+x_{i}\right)\right)\right] \\
& +G^{\prime}\left(a^{i} x_{0}+\cdots+x_{i}\right)
\end{aligned}
$$

Recalling (48), we obtain

$$
h_{1}\left(a^{i} x_{0}^{*}+\cdots+x_{i}^{*}\right)=\nu(1-a) \quad \text { for } i=0, \ldots, N-1 .
$$

Since $h_{1}$ is one-to-one we must have

$$
\begin{aligned}
x_{0}^{*} & =h_{1}^{-1}(\nu(1-a)) \\
x_{i}^{*} & =x_{0}^{*}(1-a) \text { for } i=1, \ldots, N-1 \\
x_{N}^{*} & =X_{0}-x_{0}^{*}-(N-1) x_{0}^{*}(1-a) .
\end{aligned}
$$

Note that these equations link all the trades to the initial trade $x_{0}$. Due to the dynamics (36) and (37), it follows that the process $E$ of $\xi^{*}$ is given by

$$
E_{t_{n}}=a\left(a x_{0}+x_{0}(1-a)\right)=a x_{0} .
$$

Consequently, by (46),

$$
\begin{aligned}
C^{(1)}\left(x_{0}^{*}, \ldots, x_{N}^{*}\right)= & G\left(x_{0}^{*}\right)-G(0)+(N-1)\left[G\left(a x_{0}^{*}+x_{0}^{*}(1-a)\right)-G\left(a x_{0}^{*}\right)\right] \\
& +G\left(a x_{0}^{*}+X_{0}-x_{0}^{*}-(N-1) x_{0}^{*}(1-a)\right)-G\left(x_{0}^{*} a\right) \\
= & N\left[G\left(x_{0}^{*}\right)-G\left(x_{0}^{*} a\right)\right]+G\left(X_{0}+N x_{0}^{*}(a-1)\right)-G(0) \\
=: & C_{0}^{(1)}\left(x_{0}^{*}\right) .
\end{aligned}
$$

It thus remains to minimize the function $C_{0}^{(1)}(y)$ with respect to $y$. Thanks to the existence of an optimal strategy in $\Xi$ for $C^{(1)}$, we know that $C_{0}^{(1)}(y)$ has at least one local minimum. Differentiating with respect to $y$ gives

$$
\begin{aligned}
\frac{\partial C_{0}^{(1)}(y)}{\partial y} & =N\left[F^{-1}(y)-a F^{-1}(a y)+(a-1) F^{-1}\left(X_{0}+N y(a-1)\right)\right] \\
& =N \hat{h}_{1}(y) .
\end{aligned}
$$

Lemma B.2 now implies that $C_{0}^{(1)}$ can only have one local minimum, which is also positive if it exists. This local minimum must hence be equal to $x_{0}^{*}$, which establishes both the uniqueness of the optimal strategy as well as our representation. 
Finally, it remains to prove that all market orders in the optimal strategy are strictly positive. Lemma B.2 gives $\xi_{0}^{(1)}=x_{0}^{*}>0$ and then (51) gives $\xi_{n}^{(1)}=x_{n}^{*}>0$ for $n=1, \ldots, N-1$. As for the final market order, using the facts that (53) vanishes at $y=x_{0}^{*}$ and $F^{-1}$ is strictly increasing gives

$$
\begin{aligned}
0 & =F^{-1}\left(x_{0}^{*}\right)-a F^{-1}\left(a x_{0}^{*}\right)-(1-a) F^{-1}\left(a x_{0}^{*}+x_{N}^{*}\right) \\
& >(1-a)\left[F^{-1}\left(a x_{0}^{*}\right)-F^{-1}\left(a x_{0}^{*}+x_{N}^{*}\right)\right]
\end{aligned}
$$

which in turn implies $x_{N}^{*}>0$.

\section{The optimal strategy in Model 2.}

In this section, we will minimize the function $C^{(2)}$ of (43) over the set $\Xi$ of all deterministic strategies and thereby complete the proof of Theorem 5.1. To this end, recall first that the definitions of $D$ and $E$ are given by (35)-(37). Based on their Model 2 dynamics, we will now obtain a formula of the cost function $C^{(2)}$ of (43) in terms of the functions $F, \widetilde{F}$, and $G$, where $G$ is as in (45). For any deterministic strategy $\xi=\left(x_{0}, \ldots, x_{N}\right) \in \Xi$,

$$
\begin{aligned}
C^{(2)}\left(x_{0}, \ldots, x_{N}\right) & =\sum_{n=0}^{N} \int_{D_{t_{n}}}^{D_{t_{n}}+} x f(x) d x \\
& =\sum_{n=0}^{N}\left(G\left(x_{n}+F\left(D_{t_{n}}\right)\right)-\widetilde{F}\left(D_{t_{n}}\right)\right) .
\end{aligned}
$$

We now state three technical lemmas that will allow to get the optimal strategy.

Lemma C.1 We have $C^{(2)}\left(x_{0}, \ldots, x_{N}\right) \longrightarrow+\infty$ for $|\xi| \rightarrow \infty$, and therefore there exists a local minimum of $C^{(2)}$ in $\Xi$.

Proof: We rearrange the sum in (54):

$$
\begin{aligned}
C^{(2)}\left(x_{0}, \ldots, x_{N}\right) & =\widetilde{F}\left(a F^{-1}\left(x_{N}+F\left(D_{t_{N}}\right)\right)\right) \\
& +\sum_{n=0}^{N}\left[\widetilde{F}\left(F^{-1}\left(x_{n}+F\left(D_{t_{n}}\right)\right)\right)-\widetilde{F}\left(a F^{-1}\left(x_{n}+F\left(D_{t_{n}}\right)\right)\right)\right] \\
& \geq \sum_{n=0}^{N}\left[\widetilde{F}\left(F^{-1}\left(x_{n}+F\left(D_{t_{n}}\right)\right)\right)-\widetilde{F}\left(a F^{-1}\left(x_{n}+F\left(D_{t_{n}}\right)\right)\right)\right] .
\end{aligned}
$$

For the terms in (55), we have the lower bound

$$
\widetilde{F}(z)-\widetilde{F}(a z)=\left|\int_{a z}^{z} x f(x) d x\right| \geq \frac{1}{2}\left(1-a^{2}\right) z^{2} \inf _{\widetilde{z} \in[a z, z]} f(\widetilde{z}) \geq 0 .
$$


Let

$$
H(y)=\frac{1}{2}\left(1-a^{2}\right) F^{-1}(y)^{2} \inf _{x \in\left[a F^{-1}(y), F^{-1}(y)\right]} f(x) .
$$

Then we have $H(y) \longrightarrow+\infty$ for $|y| \rightarrow \infty$, due to (20) and (14). Besides, we have

$$
C^{(2)}\left(x_{0}, \ldots, x_{N}\right) \geq H\left(\left|T_{2}(\xi)\right|_{\infty}\right)
$$

where $|\cdot|_{\infty}$ denotes again the $\ell^{\infty}$-norm on $\mathbb{R}^{N+1}$, and $T_{2}$ is the (nonlinear) transformation

$$
T_{2}(\xi)=\left(x_{0}, x_{1}+F^{-1}\left(D_{t_{1}}\right), \ldots, x_{N}+F^{-1}\left(D_{t_{N}}\right)\right) .
$$

It is sufficient to show that $\left|T_{2}(\xi)\right|_{\infty} \longrightarrow \infty$ when $|\xi| \rightarrow \infty$. To prove this, we suppose by way of contradiction that there is a sequence $\xi^{k}$ such that $\left|\xi^{k}\right|_{\infty} \longrightarrow \infty$ and $T_{2}\left(\xi^{k}\right)$ stays bounded. Then, all coordinates in the sequence $\left(T_{2}\left(\xi^{k}\right)\right)_{k}$ are bounded, and in particular $\left(x_{0}^{k}\right)_{k}$ is a bounded sequence. Therefore, $D_{t_{1}}^{k}=a F^{-1}\left(x_{0}^{k}\right)$ is also a bounded sequence. The second coordinate $x_{1}^{k}+F^{-1}\left(D_{t_{1}}^{k}\right)$ being also bounded, we get that $\left(x_{1}^{k}\right)_{k}$ is a bounded sequence. In that manner, we get that $\left(x_{n}^{k}\right)_{k}$ is a bounded sequence for any $n=0, \ldots, N$, which is the desired contradiction.

Lemma C.2 (Partial derivatives of $C^{(2)}$ ).

We have the following recursive scheme for the derivatives of $C^{(2)}\left(x_{0}, \ldots, x_{N}\right)$ for $i=0, \ldots, N-1$ :

$$
\frac{\partial}{\partial x_{i}} C^{(2)}=F^{-1}\left(x_{i}+F\left(D_{t_{i}}\right)\right)+\frac{a f\left(D_{t_{i+1}}\right)}{f\left(F^{-1}\left(x_{i}+F\left(D_{t_{i}}\right)\right)\right)}\left[\frac{\partial}{\partial x_{i+1}} C^{(2)}-D_{t_{i+1}}\right] .
$$

Proof: From (23) we get the following scheme for $D_{t_{n}}$ for a fixed $n \in\{1, \ldots, N\}$ :

$$
\begin{aligned}
& \begin{array}{cc}
D_{t_{n}} & \\
a F^{-1}\left(x_{n-1}+\right. & \left.F\left(D_{t_{n-1}}\right)\right) \\
& \|
\end{array}
\end{aligned}
$$

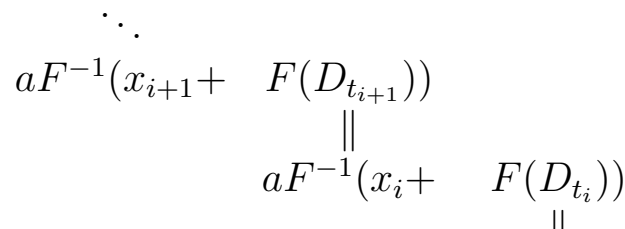

$$
\begin{aligned}
& \ddots \\
& a F^{-1}\left(x_{0}\right) .
\end{aligned}
$$

Therefore the following relation holds for the partial derivatives of $D_{t_{n}}$ :

$$
\frac{\partial}{\partial x_{i}} D_{t_{n}}=\frac{a f\left(D_{t_{i+1}}\right)}{f\left(F^{-1}\left(x_{i}+F\left(D_{t_{i}}\right)\right)\right)} \frac{\partial}{\partial x_{i+1}} D_{t_{n}}, \quad i=0, \ldots, n-2 .
$$


Furthermore, according to (54) and (48),

$$
\begin{aligned}
\frac{\partial}{\partial x_{i}} C^{(2)}=F^{-1} & \left(x_{i}+F\left(D_{t_{i}}\right)\right)+ \\
& +\sum_{n=i+1}^{N} f\left(D_{t_{n}}\right) \frac{\partial}{\partial x_{i}} D_{t_{n}}\left[F^{-1}\left(x_{n}+F\left(D_{t_{n}}\right)\right)-D_{t_{n}}\right]
\end{aligned}
$$

for $i=0, \ldots, N$. Combining (58) and (57) yields (56). Note that (57) is only valid up to $i=n-2$.

Lemma C.3 Under the assumptions of the Theorem 5.1, equation (21) has at most one solution $x_{0}>0$. Besides, the function $g(x):=f(x)-a f(a x)$ is positive.

Proof: Uniqueness will follow if we can show that both $h_{2} \circ F^{-1}$ and

$$
\hat{h}_{2}(y):=-F^{-1}\left(X_{0}-N\left[y-F\left(a F^{-1}(y)\right)\right]\right)
$$

are strictly increasing. Moreover, $h_{2} \circ F^{-1}(0)=0$ and $\hat{h}_{2}(0)<0$ so that any zero of $h_{2} \circ F^{-1}+\hat{h}_{2}$ must be strictly positive.

The function $h_{2}$ is one-to-one, has zero as fixed point, and satisfies (26). It is therefore strictly increasing, and since $F^{-1}$ is also strictly increasing, we get that $h_{2} \circ F^{-1}$ is strictly increasing. It remains to show that $\hat{h}_{2}$ is strictly increasing. We have that

$$
\hat{h}_{2}^{\prime}(y)=N \frac{f\left(F^{-1}(y)\right)-a f\left(a F^{-1}(y)\right)}{f\left(F^{-1}(y)\right) f\left(F^{-1}\left(X_{0}-N\left[y-F\left(a F^{-1}(y)\right)\right]\right)\right)},
$$

is strictly positive, because, as we will show now, the numerator of this term is positive. The numerator can be expressed as $g\left(F^{-1}(y)\right)$ for $g$ as in the assertion. Hence, establishing strict positivity of $g$ will conclude the proof. To prove this we also define $g_{2}(x):=f(x)-a^{2} f(a x)$ so that

$$
h_{2}(x)=x \frac{g_{2}(x)}{g(x)} .
$$

Both functions $g$ and $g_{2}$ are continuous and have the same sign for all $x \in \mathbb{R}$ due to the properties of $h_{2}$ explained at the beginning of this proof. Because of $g(x)<g_{2}(x)$ for all $x \in \mathbb{R}$, we infer that there can be no change of signs, i.e., either $g(x)>0$ and $g_{2}(x)>0$ for all $x \in \mathbb{R}$ or $g(x)<0$ and $g_{2}(x)<0$ everywhere. With $g(0)=f(0)(1-a)>0$ we obtain the positivity of $g$.

Theorem 5.1 will now follow by combining the following proposition with the arguments explained in Section A.

Proposition C.4 The function $C^{(2)}: \Xi \rightarrow \mathbb{R}$ has the strategy $\xi^{(2)}$ from Theorem 5.1 as its unique minimizer. Moreover, the components of $\xi^{(2)}$ are all strictly positive. 
Proof: The structure of the proof is similar to the one of Theorem4.1 although the computations are different. Thanks to Lemma C.1, we know that there exists an optimal strategy $\xi^{*}=$ $\left(x_{0}^{*}, \ldots, x_{N}^{*}\right) \in \Xi$. There also exists a corresponding Lagrange multiplier $\nu$ such that

$$
\frac{\partial}{\partial x_{i}} C^{(2)}\left(x_{0}^{*}, \ldots, x_{N}^{*}\right)=\nu, \quad i=0, \ldots, N .
$$

From (56), we get

$$
\nu=h_{2}\left(F^{-1}\left(x_{i}^{*}+F\left(D_{t_{i}}\right)\right)\right), \quad i=0, \ldots, N-1 .
$$

Since $h_{2}$ is one-to one, this implies in particular that $x_{i}^{*}+F\left(D_{t_{i}}\right)$ does not depend on $i=$ $0, \ldots, N-1$. It follows from (23) also $D_{t_{i}+}=F^{-1}\left(x_{i}^{*}+F\left(D_{t_{i}}\right)\right)$ is constant in $i$, and so

$$
D_{t_{i}+}=D_{t_{0}+}=F^{-1}\left(x_{0}^{*}\right) \quad \text { and } \quad D_{t_{i+1}}=a F^{-1}\left(x_{0}^{*}\right) \text {. }
$$

Hence,

$$
\begin{aligned}
x_{0}^{*} & =F\left(h_{2}^{-1}(\nu)\right), \\
x_{i}^{*} & =x_{0}^{*}-F\left(D_{t_{i}}\right)=x_{0}^{*}-F\left(a F^{-1}\left(x_{0}^{*}\right)\right) \quad \text { for } i=1, \ldots, N-1, \\
x_{N}^{*} & =X_{0}^{*}-x_{0}^{*}-(N-1)\left[x_{0}^{*}-F\left(a F^{-1}\left(x_{0}^{*}\right)\right)\right] .
\end{aligned}
$$

These equations link all market orders to the initial trade $x_{0}^{*}$. Using (60) and once again (59)), we find that $C^{(2)}\left(x_{0}^{*}, \ldots, x_{N}^{*}\right)$ is equal to

$$
\begin{aligned}
C_{0}^{(2)}\left(x_{0}^{*}\right) & :=C^{(2)}\left(x_{0}^{*}, x_{0}^{*}-F\left(a F^{-1}\left(x_{0}^{*}\right)\right), \ldots, X_{0}-N x_{0}^{*}+(N-1) F\left(a F^{-1}\left(x_{0}^{*}\right)\right)\right) \\
& =N\left[G\left(x_{0}^{*}\right)-\widetilde{F}\left(a F^{-1}\left(x_{0}^{*}\right)\right)\right]+G\left(X_{0}+N\left[F\left(a F^{-1}\left(x_{0}^{*}\right)\right)-x_{0}^{*}\right]\right) .
\end{aligned}
$$

The initial trade $x_{0}^{*}$ must clearly be a local minimum of $C_{0}^{(2)}$ and thus $\frac{\partial}{\partial y} C_{0}^{(2)}\left(x_{0}^{*}\right)=0$. Therefore,

$$
0=N\left[D_{0+}-a^{2} D_{0+} \frac{f\left(D_{t_{1}}\right)}{f\left(D_{0+}\right)}+D_{t_{N}}\left(a \frac{f\left(D_{t_{1}}\right)}{f\left(D_{0+}\right)}-1\right)\right],
$$

which is equivalent to

$$
D_{t_{N}+}=D_{0+} \frac{f\left(D_{0+}\right)-a^{2} f\left(D_{t_{1}}\right)}{f\left(D_{0+}\right)-a f\left(D_{t_{1}}\right)} .
$$

This is just equation (21), which has at most one solution, due to Lemma C.3. This concludes the proof of the existence and the representation of the optimal strategy $\xi^{(2)}$ in Theorem 5.1 .

Finally, we need to show the strict positivity of the optimal strategy. Thanks to the positivity of the optimal $x_{0}^{*}$, we get

$$
x_{i}^{*}=x_{0}^{*}-F\left(a F^{-1}\left(x_{0}^{*}\right)\right)>0
$$

for $i=1, \ldots, N-1$. So it only remains to show that $x_{N}^{*}>0$. We infer from (61) and (59) that

$$
D_{t_{N}+}=D_{0+} \frac{f\left(D_{0+}\right)-a^{2} f\left(a D_{0+}\right)}{f\left(D_{0+}\right)-a f\left(a D_{0+}\right)}=D_{0+}\left[1+\frac{a f\left(a D_{0+}\right)-a^{2} f\left(a D_{0+}\right)}{f\left(D_{0+}\right)-a f\left(a D_{0+}\right)}\right] \text {. }
$$

The fraction on the right is strictly positive due to Lemma C.3. Hence,

$$
D_{t_{N}+}>D_{0+}=\frac{1}{a} D_{t_{N}}>D_{t_{N}}
$$

which implies $x_{N}^{*}>0$. 


\section{Optimal strategy for block-shaped LOB.}

Here we prove the results of Section 6 ,

Our aim is to prove Proposition 6.2, i.e., to show that the strategy (27) satisfies the recursion (31). The key point is that we have indeed explicit formulas for the coefficients in the backward schemes of Proposition 6.2.

Lemma D.1 The coefficients $\alpha_{n}, \beta_{n}$, and $\gamma_{n}$ from (33) are explicitly given by

$$
\begin{aligned}
\alpha_{n} & =\frac{\left(1+a^{-1}\right)-q \lambda\left[(N-n)\left(a^{-1}-1\right)+2\left(1+a^{-1}\right)\right]}{2 q\left[(N-n)\left(a^{-1}-1\right)+\left(1+a^{-1}\right)\right]} \\
\beta_{n} & =\frac{1+a^{-1}}{\left[(N-n)\left(a^{-1}-1\right)+\left(1+a^{-1}\right)\right]} \\
\gamma_{n} & =\frac{(N-n)\left(1-a^{-1}\right)}{2 \kappa\left[(N-n)\left(a^{-1}-1\right)+\left(1+a^{-1}\right)\right]} .
\end{aligned}
$$

The explicit form of the sequences $\delta_{n}, \epsilon_{n}$ and $\phi_{n}$ from (32) is

$$
\begin{aligned}
\delta_{n} & =\frac{2 a^{-2}\left[(N-n)\left(a^{-1}-1\right)+\left(1+a^{-1}\right)\right]}{\kappa\left[(N-n)\left(1-a^{-2}\right)+(N-n+2)\left(a^{-3}-a^{-1}\right)\right]} \\
\epsilon_{n} & =\frac{\kappa\left(a^{-1}-a\right)}{\left[(N-n)\left(a^{-1}-1\right)+\left(1+a^{-1}\right)\right]} \\
\phi_{n} & =\frac{(N-n+1)\left(a^{-1}-a\right)-(N-n)\left(1-a^{2}\right)}{\left[(N-n)\left(a^{-1}-1\right)+\left(1+a^{-1}\right)\right]} .
\end{aligned}
$$

This Lemma can be proved in two steps. First, by a backward induction, we get the explicit formulas for $\alpha, \beta$ and $\gamma$. Then, combining (62) with (33) and (32), we get (63).

Proof of Proposition 6.2. We can deduce the following formulas from the preceding lemma:

$$
\delta_{n} \epsilon_{n}=\frac{2}{(N-n)(1-a)+2}, \quad \delta_{n} \phi_{n}=\frac{2}{\kappa} \frac{(N-n)(1-a)+1}{(N-n)(1-a)+2} .
$$

They will turn out to be convenient in (31).

Let us now consider the optimal strategy $\left(\xi_{0}^{*}, \ldots, \xi_{N}^{*}\right)$ from (27). We consider the associated processes $D_{t}:=D_{t}^{A}$ and $X_{t}$ as defined in (28) and (30). For $n=0$, we have

$$
\xi_{0}^{*}=\frac{X_{0}}{(N-1)(1-a)+2}=\frac{1}{2} \delta_{1} \epsilon_{1}
$$

and it satisfies (31) because $D_{0}=0$. For $n \geq 1$, we can show easily by induction on $n$ that $D_{t_{n}}=a \kappa \xi_{0}^{*}$. From (27), we get that $\xi_{n}^{*}=(1-a) \xi_{0}^{*}$ for $n \notin\{0, N\}$, and therefore we get

$$
X_{t_{n}}=X_{0}-\xi_{0}^{*}-(n-1)(1-a) \xi_{0}^{*}=[(N-n)(1-a)+1] \xi_{0}^{*} .
$$

Using these formulas, and combining with (64), it is now easy to check that for $n \in\{1, \ldots, N-1\}$,

$$
\xi_{n}^{*}=\frac{1}{2}\left[\delta_{n+1} \epsilon_{n+1} X_{t_{n}}-\delta_{n+1} \phi_{n+1} D_{t_{n}}\right],
$$

which shows that the optimal strategy given in (27) solves (31). 
Acknowledgement. Support from the Deutsche Bank Quantitative Products Laboratory is gratefully acknowledged. The authors thank the Quantitative Products Group of Deutsche Bank, in particular Marcus Overhaus, Hans Bühler, Andy Ferraris, Alexander Gerko, and Chrif Youssfi for stimulating discussions and useful comments (the statements in this paper, however, express the private opinion of the authors and do not necessarily reflect the views of Deutsche Bank). Moreover, it is a pleasure to thank Anna Obizhaeva and Torsten Schöneborn for helpful comments on earlier versions of this paper.

\section{References}

[1] Alfonsi, A., Fruth, A., Schied, A. Constrained portfolio liquidation in a limit order book model. Banach Center Publ. 83, 9-25 (2008).

[2] Almgren, R. Optimal execution with nonlinear impact functions and trading-enhanced risk, Applied Mathematical Finance 10 , 1-18 (2003).

[3] Almgren, R., Chriss, N. Value under liquidation. Risk, Dec. 1999.

[4] Almgren, R., Chriss, N. Optimal execution of portfolio transactions. J. Risk 3, 5-39 (2000).

[5] Almgren, R., Lorenz, J. Adaptive arrival price. In: Algorithmic Trading III: Precision, Control, Execution, Brian R. Bruce, editor, Institutional Investor Journals (2007).

[6] Almgren, R., Thum, C. Hauptmann, E., Li, E. Equity market impact. Risk, July (2005).

[7] Bank, P., Baum, D. Hedging and portfolio optimization in financial markets with a large trader. Math. Finance 14, no. 1, 1-18 (2004).

[8] Bertsimas, D., Lo, A. Optimal control of execution costs. Journal of Financial Markets, 1, 1-50 (1998).

[9] Biais, B., Hillion, P., Spatt, C. An empirical analysis of the limit order book and order flow in Paris Bourse. Journal of Finance 50, 1655-1689 (1995).

[10] Bouchaud, J. P., Gefen, Y., Potters, M. , Wyart, M. Fluctuations and response in financial markets: the subtle nature of 'random' price changes. Quantitative Finance 4, 176 (2004).

[11] Brunnermeier, M., Pedersen, L. Predatory trading. Journal of Finance 60, 1825-1863 (2005).

[12] Carlin, B., Lobo, M., Viswanathan, S. Episodic liquidity crises: Cooperative and predatory trading. Forthcoming in Journal of Finance.

[13] Cetin, U., Jarrow, R., Protter, P. Liquidity risk and arbitrage pricing theory. Finance Stoch. 8 , no. 3, 311-341 (2004). 
[14] Frey, R. Derivative asset analysis in models with level-dependent and stochastic volatility. Mathematics of finance, Part II. CWI Quarterly 10 , no. 1, 1-34 (1997).

[15] Frey, R., Patie, P. Risk management for derivatives in illiquid markets: a simulation study. Advances in finance and stochastics, 137-159, Springer, Berlin, 2002.

[16] Obizhaeva, A., Wang, J. Optimal Trading Strategy and Supply/Demand Dynamics, Preprint, forthcoming in Journal of Financial Markets.

[17] Potters, M., Bouchaud, J.-P. More statistical properties of order books and price impact. Physica A 324, No. 1-2, 133-140 (2003).

[18] Schied, A., Schöneborn, T. Optimal basket liquidation with finite time horizon for CARA investors. Preprint, TU Berlin (2008).

[19] Schied, A., Schöneborn, T. Risk aversion and the dynamics of optimal liquidation strategies in illiquid markets. To appear in Finance and Stochastics.

[20] Schöneborn, T., Schied, A. Competing players in illiquid markets: predatory trading vs. liquidity provision. Preprint, TU Berlin.

[21] Weber, P., Rosenow, B. Order book approach to price impact. Quantitative Finance 5, no. 4, 357-364 (2005). 